\title{
Exploring experienced nurses' views, attitudes and expectations of graduate nurses in the operating theatre
}

Follow this and additional works at: https://www.journal.acorn.org.au/jpn

Part of the Health Services Administration Commons, Health Services Research Commons, Perioperative, Operating Room and Surgical Nursing Commons, and the Surgery Commons

(c) (i)

This work is licensed under a Creative Commons Attribution 4.0 License.

\section{Recommended Citation}

Freeling, Michelle; Parker, Steve; and Breaden, Katrina (2017) "Exploring experienced nurses' views, attitudes and expectations of graduate nurses in the operating theatre," Journal of Perioperative Nursing: Vol. 30 : Iss. 1 , Article 2.

Available at: https://doi.org/10.26550/2209-1092.1012

https://www.journal.acorn.org.au/jpn/vol30/iss1/2

This Article is brought to you for free and open access by Journal of Perioperative Nursing. It has been accepted for inclusion in Journal of Perioperative Nursing by an authorized editor of Journal of Perioperative Nursing. 


\section{Author \\ Michelle Freeling \\ BN (Hons), RN \\ School of Nursing and Midwifery \\ The Flinders University of South Australia \\ Dr Steve Parker \\ PhD, BEd, DipT (Nurse Ed), RN, RPN \\ Associate Dean (Teaching \& Learning) \\ Exploring experienced nurses' views, attitudes and expectations of graduate nurses in the operating theatre}

School of Nursing and Midwifery

The Flinders University of South Australia

Dr Katrina Breaden

PhD, MN, Grad Cert Ed (Higher Education), RN

Lecturer in Nursing

School of Nursing and Midwifery

The Flinders University of South Australia

\section{Corresponding author}

Michelle Freeling

BN (Hons), RN

School of Nursing and Midwifery

The Flinders University of South Australia

While the new graduate nurse journey has been well documented, little research exists from the perspective of the experienced nurse, particularly in the perioperative environment. Barriers to graduate nurse support need to be understood, as job satisfaction, nurse wellbeing and quality of patient care can be negatively affected.

\section{Background}

Throughout the history of nursing, the relationships between experienced nurses and nurses undergoing training have been widely discussed. There is a plethora of research which examines the graduate nurse journey and subsequent feelings and attitudes towards workplace integration ${ }^{1-7}$. However, little research exists regarding the view of the experienced registered nurse concerning graduate nurses. Experienced registered nurses significantly contribute to the development of the graduate nurse, and it is widely recognised that experienced registered nurses acting as preceptors is essential to graduate nurse development ${ }^{8-10}$.

Perioperative nursing, defined as providing care for patients in the period prior, during and following surgery or interventional procedures ${ }^{11}$, presents further challenges for novices. The intrinsic stress of undertaking surgical procedures, the absence of experienced staff, the seclusion and constraint of the operating room and the high patient acuity contribute to the challenging nature of the perioperative environment for graduate nurses ${ }^{12}$. Perioperative nursing requires the ability to perform an abundance of clinical skills in a technically complex environment ${ }^{13}$, and nurses are rarely offered meaningful perioperative training in undergraduate education programs ${ }^{14}$. These challenging characteristics of perioperative nursing may cause new graduate nurses in the operating theatre to feel isolated and discouraged ${ }^{13}$.

Additionally, negative staff attitudes have been highlighted as one of the most powerful barriers to graduate nurse support ${ }^{10}$, further compounding the challenges of the perioperative graduate nurse journey. It has been noted that a negative approach from senior nurses towards graduate nurses creates a barrier for optimal skill utilisation by the graduate ${ }^{15}$. Nursing culture has been perceived, both historically and contemporarily, as negative ${ }^{16-18}$ and many graduate nurses find it challenging to become accepted in the culture of the unit ${ }^{19}$. The unique challenges of perioperative nursing and the new graduate nurse journey, coupled with the present climate of budgetary constraints in health care, mean that investigating barriers in nursing is vital.

A literature review conducted as part of this study revealed a lack of research investigating experienced nurses' views, attitudes and 
expectations of graduate nurses in any setting ${ }^{19}$. A total of ten studies were reviewed ${ }^{15,18,19,21-27}$ and four main themes were identified:

1. experienced nurses believe graduate nurses are inadequately prepared for practice

2. graduates need to improve practical nursing skills

3. experienced nurses have negative attitudes towards graduates

4. experienced nurses believe graduate nurses lack the confidence necessary to practice competently.

This literature review demonstrated a significant lack of research on this topic and a need for further investigation. To enable graduates to fulfil their potential, the attitudes, beliefs and expectations of experienced registered nurses and graduate nurses must be explored with regards to graduate nurses entering the workforce. Only then can these attitudes and feelings be addressed and methods put in place to facilitate good relationships between graduate nurses and experienced registered nurses. This understanding may ultimately allow graduate nurses to utilise their full range of skills, practice confidently and comfortably and become a valuable part of the health care team.

The above considerations culminated in the research question: what are experienced nurses' views, attitudes and expectations of graduate nurses in the operating theatre? The objective of this research is to unlock the experiences and opinions of experienced operating theatre nurses regarding graduate nurses and, in doing so, explore the relationship between experienced and graduate nurses as well as revealing what experienced operating theatre nurses underwent as graduate nurses. This research question aims to give nurses a voice and allow readers of this study a glimpse of theatre life that describes the nurses' world within this particular context.

\section{Methods}

\section{Aim and design}

The aim of this study was to explore experienced nurses' views about, attitudes towards and expectations of graduate nurses in the operating theatre. Data collection was undertaken using face-to-face, unstructured, in-depth interviews. An interpretive hermeneutic approach was used, allowing interpretation of the data and eliciting meaning from the participants' stories.

\section{Settings and participants}

Participants were recruited from the perioperative department of a busy metropolitan teaching hospital. A convenience sample of nurses with more than three years' experience in the operating theatre who were currently working or had previously worked directly with graduate nurses were invited to participate. These criteria ensured participants were experienced and competent perioperative nurses who were capable of reflecting on their overall views about, attitudes towards and expectations of graduate nurses.

\section{Data collection}

Ethics approval was given by the appropriate Human Research Ethics Committee (506.14) and the sitespecific ethics committee. Informed consent was gained before the interviews. Participants consisted of a convenience sample of three perioperative nurses and were recruited for a 45-minute, in-depth interview with the aim of exploring their views about, attitudes towards and expectations of graduate nurses in the operating theatre of a large metropolitan tertiary teaching hospital in Adelaide, South Australia. The nurses had five, nine and more than twenty years' experience respectively. All participants had experience teaching and working with graduate nurses in the perioperative setting. The in-depth method of interviewing deemed necessary by a hermeneutic methodology allows meaning to be explored and details of participants' real-world experiences to be considered.

\section{Data analysis}

The primary researcher immediately transcribed verbatim each interview after it occurred. The researcher listened to the recorded interview several times while reading the transcript. Central concerns, important themes or meanings unfolded for particular participants and were highlighted during this step by the researcher ${ }^{28}$. The researcher read and analysed the transcript again, this time with the intention of deriving themes from the data. Summaries of central themes were developed based on the researchers' initial interpretations. During this process, the transcripts were simultaneously examined with the emerging interpretation. NVivo software was used to manage and code the data.

\section{Findings and discussion}

Application of the data analysis techniques applied to the interview data resulted in six predominant themes, and concepts within these themes (Table 1). Some of the issues that arose are briefly discussed below; quotations (using participant numbers to identify each) are used to illustrate the findings.

\section{The importance of mentoring}

In this study, mentoring emerged as a significant subconcept within the concept of education and theme of people. Participants described their 


\begin{tabular}{|c|c|c|}
\hline $\begin{array}{l}\text { Themes } \\
\text { (in order of } \\
\text { prevalence) }\end{array}$ & Concept & Subconcept \\
\hline \multirow[t]{7}{*}{ 1. People } & 1.1 Personalities & \\
\hline & \multirow[t]{5}{*}{ 1.2 Education } & 1.2.1 Learning \\
\hline & & 1.2.2 Attitudes \\
\hline & & 1.2.3 Workload \\
\hline & & 1.2.4 Continuity \\
\hline & & 1.2.5 Mentoring \\
\hline & 1.3 Management & \\
\hline \multirow[t]{3}{*}{ 2. Theatre } & 2.1 Patient-centered care & \\
\hline & 2.2 Roles & \\
\hline & 2.3 Unfamiliarity & \\
\hline \multirow[t]{2}{*}{ 3. Structure } & 3.1 Specialties & \\
\hline & 3.2 Allocations & \\
\hline 4. Culture & 4.1 Generations & \\
\hline \multirow[t]{4}{*}{ 5. Graduates } & 5.1 Thriving & \\
\hline & 5.2 Experience & \\
\hline & 5.3 Overconfidence & \\
\hline & 5.4 The basics & \\
\hline 6. Stress & & \\
\hline
\end{tabular}

Table 1: Themes, concepts and subconcepts

experiences of receiving mentoring as graduate nurses and highlighted the importance of guidance. Graduates may thrive if a senior nurse notices them and chooses to form a supportive, mentoring relationship. The participants declared mentoring an essential part of an effective relationship between experienced nurses and graduate nurses in the operating theatre. Similarly, Lydon and Burke ${ }^{29}$ found that a supportive preceptor enhanced learning whereas an unsupportive preceptor impeded learning. In this study, participants uncovered a current lack of nurturing and mentoring in their workplace, which is, unfortunately, inhibiting graduate nurse success in this environment:

I was nurtured so well; I think that's something that's lacking at the moment. And, you know, from the bigger picture I think it's obviously time and money constraints. Public health is under stress. Everything is bare bones. And so we are losing good people because of it, because they're not being supported and mentored and nurtured (N1).

As mentoring promotes a supportive theatre learning environment ${ }^{30,31}$, this lack is worrying. Education regarding the ramifications of neglecting a graduate nurse's learning opportunities, the potential presence of horizontal violence and failure to integrate into the perioperative team should be given to all relevant individuals in the workplace.

\section{Unfamiliar environment}

The 'foreign' nature of the operating theatre presents a unique challenge to graduate nurses and unfamiliarity emerged as a significant concept within the theatre theme. Experienced nurses conveyed the atypical nature of operating theatre nursing, and that graduate nurses were initially unfamiliar with the nursing skills required:

Ward nursing is more comfortable for graduates because that's where their three years of clinical placements were. You have to be a lot more flexible [in the operating theatre] than on the ward (N1).

Experienced nurses conveyed that different skills and different flexibility are necessary for theatre nursing.

You know, we have so many intricacies of which way to hand an instrument, for example upside down, left to right, forward, backwards - and if you do it incorrectly ... You're always going to at the beginning but at some point there's an expectation you'll pick it up. And surgeons definitely let you know if you're not doing it right, either in a nice way or a mean way (N1).

Due to the absence of perioperative nursing instruction in most undergraduate programs, the core processes and atmosphere in the operating theatre is foreign to most graduate nurses ${ }^{32}$. Another participant discussed the importance of emphasising patient-centred care in the operating theatre:

In theatre when you first start as a graduate it is not exactly patientcentred. You're not there hands on with the patient taking blood pressure, asking them how their pain is, because you're opening a sterile packet for someone wearing a gown behind a trolley. At the beginning typically you're so overwhelmed, you're not even thinking about the patient... (N1).

Due to the unfamiliar nature of theatre nursing, participants 
asserted that previous theatre experience was beneficial to nurses beginning a perioperative graduate year. Unfortunately, perioperative nursing is often under-utilised for undergraduate clinical placement experience ${ }^{33}$. Baumberger-Hen ry ${ }^{19}$ found that material required to work competently in a specialised area is often not taught as part of undergraduate education. However, a perioperative placement (with instructional guidance from a placement coordinator) may allow the student to develop a greater understanding of surgical ward nursing than surgical ward nursing experience alone ${ }^{34}$. Reallocating a portion of time and resources from surgical nursing to perioperative nursing may ease the difficulty that universities are having in securing placements and would improve recruitment to perioperative nursing ${ }^{34}$.

\section{Length of time in rotations}

The theme of 'structure' was the third in prevalence and included two concepts - specialties and allocations. This study uncovered the detrimental effect on theatre teams of shorter specialty rotations during the graduate nurse year. It became apparent that experienced nurses are hesitant to invest time and energy in graduate nurses' education if they are aware the graduate nurse will soon be moving to another specialty. Participants described the frustration of continually teaching graduate nurses. According to Young ${ }^{35}$, perioperative nurses are overworked and understaffed so training a high volume of new nurses as well as meeting the daily demands of theatre nursing increases the pressure nurses face. Indeed, in this study, minimal surgical rotations of three months were described as weakening the relationships within theatre teams.

We're struggling with that at the moment, because our current nurses are doing three-month rotations - sometimes six if they're really struggling but that's the only time it's extended, if they're struggling, and it's affecting the relationships. People are deskilling quicker because they're churned through like a production line (N1).

A better understanding of underlying pathology, rationales behind surgical techniques and nursing methods may be developed through longer rotations. This may then have a positive impact on graduate nurse performance in future rotations. Similarly, Pupkiewicz, Kitson and Perry ${ }^{36}$ reported a need for graduate nurses to stay in one rotation until they achieve clinical competence. Graduate nurses may feel overwhelmed if they change specialties too soon, due to the increase in volume of information ${ }^{36}$. Overall, longer surgical rotations for graduate nurses will increase trust between team members, allow a better understanding of specific procedures and prevent the volume of information from becoming overwhelming.

\section{Generational differences}

Tension between older and younger nurses arose as a significant source of distress for participants in this study making 'generations' a significant concept under the theme of 'Culture'. Many nurses continued to treat the theatre environment as in the past - where surgeons were allocated one scrub nurse and surgical specialties had exclusive teams:
A lot of the nurses will treat it like 'the old days' where they would have one scrub nurse or they try to keep the specialties very exclusive... Although we've got all the skills in the world and we're extremely competent, they still won't relax and they still won't be accepting of you. It's because of the attitudes that the older nursing generation are perpetuating... (N1).

The friction between the older and younger nurses in the theatre environment is a significant cause of horizontal violence, a contentious issue in nursing today. The negative views of graduate nurses held by experienced nurses significantly affect their investment and effort regarding the provision of mentoring and education. This intra-professional tension is of substantial concern as disruptive nurse relationships may affect optimal graduate nurse training, inhibit communication within the health care team and ultimately affect the retention of graduate nurses ${ }^{37}$. Further research is needed to investigate relationships between perioperative nurses of different generations.

\section{Occupational stress}

Stress - a significant issue in perioperative nursing - was an underlying issue for all participants. One participant discussed occupational stress:

The operating theatre environment can become quite stressful if graduate nurses don't know the severity of the situation and then things can escalate to the point where they can't manage...you need to know when to ask for help (N3).

Stress is also experienced if trust, positive working relationships and familiarity between members of the theatre team are absent. Another participant stated:

The reason a theatre works is the relationship and the trust you have [between the] scrub and scout, between the surgeon and nurses and the team. If it's a team they [surgeons] don't know, it's instantly stressful (N1).

A recent review of the occupational stress of scrub/scout practitioners revealed that occupational stress in the operating theatre impacts on perioperative nurses' health and behaviours. Jacob ${ }^{38}$ discovered factors that increase the occupational 
stress experienced by scrub/scout nurses include the atypical nature of theatre nursing work, lack of resources, work-related stress and surgeon-perpetuated bullying. Occupational stress can increase perioperative nurses' engagement with unhealthy coping behaviours such as smoking, overeating and increased alcohol consumption ${ }^{39,40}$. Further, occupational stress may lead to fatigue, reduced concentration and increased sick leave, which may affect patient safety ${ }^{38}$. The findings in this study further extend this work by directly identifying factors that influence stress in the perioperative environment. Stress resulted from numerous and, often, cumulative factors, including bullying behaviours, increased workload, generational differences, concerns with confidence and the unfamiliarity of the perioperative setting.

\section{Bullying behaviours}

Although not identified as a major theme, workplace bullying is not uncommon in the perioperative environment and contributes to occupational stress. Horizontal violence (nurse-to-nurse violence) and surgeon-perpetuated, or surgeon-to-nurse, violence were established as significant issues in this study. Surgeon-perpetuated violence may be psychological or physical. Psychological abuse includes belittling, yelling, condescension and ignoring. Physical abuse includes shoving, hitting, throwing instruments and kicking ${ }^{41}$. In a study exploring the incidence and impact of physician verbal abuse on perioperative nurses, Cook et al. ${ }^{42}$ reported that 91 per cent of nurse participants had experienced some verbal abuse from physicians in the past year. Similarly, participants in the current study identified that surgeon-perpetuated violence detrimentally affected their experiences in the operating theatre. One participant tells of her experience:
In neurosurgery I remember being told [by a senior nurse] to give these new forceps to the surgeon, who was notorious for being horrible to staff. And she's, like, 'he'll be really happy, we specially ordered these'. I handed them to him at the beginning of the case, he threw them across the room, looked at me and said 'don't ever hand them to me again' (N1).

Behaviours such as verbal abuse, throwing surgical instruments and belittling staff, and the reputation of particular surgeons for exhibiting negative behaviours compounded nurses' occupational stress. Factors that put nurses at risk of experiencing abuse from surgeons include personality differences, closeness of working relationships, stressful patient care situations and unequal power relationships ${ }^{42}$. Graduate nurses are particularly vulnerable to abuse, as they may not understand the surgeon's routine or preferences ${ }^{41}$. Abuse exacerbates the stress and confusion already experienced when new to the perioperative environment and results in adverse psychological, physical and social effects.

Workplace violence poses a serious threat to the health and wellbeing of nurses, and the World Health Organization (WHO) has therefore stated that elimination of these behaviours is a high priority ${ }^{43}$. A victim of workplace violence may experience insomnia, low self-esteem, low work morale, disconnection from others, depression, and increased sick leave ${ }^{44}$. Graduate nurses may experience burnout and disempowerment because of bullying, which may lead to leaving the profession altogether ${ }^{37}$. As the retention of new graduates is essential for a sustainable nursing workforce, it is critical to address horizontal violence and create positive working environments. Further research is needed to investigate the effects of interand intra-professional abuse on graduate nurses in the perioperative environment and explore if formal communication and assertiveness training would enable graduate nurses to cope with abusive situations.

\section{Limitations}

This study was undertaken as part of the researchers' honours year so time restraints were a significant constraint. As this study documents the opinions and experiences of three nurses, it is crucial to avoid generalising these findings to all nurses. This study should be repeated with more participants to allow data saturation to be reached. However, the participants' words are still worthy of consideration and some interesting themes are put forth in this paper. Also, participants were only recruited from one institution and a multisite study would reduce the effect of specific local factors particular to one setting ${ }^{45}$.

\section{Conclusions}

This study has investigated nurses' views about, attitudes towards and expectations of graduate nurses in the operating theatre of a busy metropolitan hospital. This issue is significant as graduate nurses face many challenges when entering the workforce and there is little research exploring experienced registered nurses' views about, attitudes towards and expectations of graduate nurses in the operating theatre. As negative staff attitudes have been highlighted as one of the most powerful barriers to graduate nurse support, considering the origin of these attitudes along with the expectations and views of experienced registered nurses is essential.

The participants' views about, attitudes towards and expectations of graduate nurses in the operating theatre were explored in this study and the process of undertaking this study was a worthwhile journey of discovery. Recommendations for 
education and future research were made. Nursing is a profession that has been perceived, both historically and contemporarily, as 'eating its young' and this study highlights factors which may prevent positive relationships between experienced nurses and graduate nurses.

\section{References}

1. Pellico LH, Brewer CS, Kovner CT. What newly licensed registered nurses have to say about their first experiences. Nurs Outlook 2009;7(4):194-203.

2. Wangensteen $D$, Johansson IS, Nordström $\mathrm{G}$. The first year as a graduate nurse - An experience of growth and development. J Clin Nurs 2008;17(14):1877-1885.

3. Halfer D, Graf E. Graduate nurse perceptions of the work experience. Nurs Econ 2006;24(3):150-157.

4. McKenna LG, Green C. Experiences and learning during a graduate nurse program: An examination using a focus group approach. Nurse Educ Pract 2004;4(4):258263.

5. Malouf N, West S. Fitting in: A pervasive new graduate nurse need. Nurs Educ Today 2011;31(5):488-493.

6. Cubit KA, Ryan B. Tailoring a graduate nurse program to meet the needs of our next generation nurses. Nurs Educ Today 2011;31(1):65-71.

7. Little JP, Ditmer D, Bashaw MA. New graduate nurse residency: A network approach. J Nurs Adm 2013;43(6):361-366.

8. Sayers JM, DiGiacomo M, Davidson PM. The nurse educator role in the acute care setting in Australia: Important but poorly described. Aust J Adv Nurs 2011;28(4):44-83.

9. Giallonardo LM, Wong CA. Authentic leadership of preceptors: Predictor of new graduate nurses' work engagement and job satisfaction. J Nurs Manag 2010;18(8):9931003.

10. Johnstone MJ, Kanitsake O, Currie T. The nature and implications of support in graduate nurse transition programs: An Australian study. J Prof Nurs 2008;28(1)46-53.

11. Australian College of Operating Room Nurses. Standards for Perioperative Nursing in Australia $14^{\text {th }}$ Ed. Adelaide, South Australia: ACORN; 2016.

12. Bigony L, Lipke TG, Lundberg A, McGraw $\mathrm{CA}$, Pagac GL, Rogers A. Lateral violence in the perioperative setting. AORN J 2009;89(4):688-700

13. Persaud D. Mentoring the new graduate perioperative nurse: A valuable retention strategy. AORN J 2008;87(6):1173-1179.

14. Stobinski J. Preoperative nursing competency. AORN Journal 2008;88(3): 417-418, 421-426, 428-430, 432, 434-436.
15. Bircumshaw D. A survey of the attitudes of senior nurses towards graduate nurses. J Adv Nurs 1989;14(1):68-72.

16. Meissner JE. Nurses: Are we eating our young? Nursing 1986;16(3):51-53.

17. Stanley KM, Dulaney P, Martin MM. Nurses 'eating our young' - it has a name: Lateral violence. S C Nurse 2007;14(1):17-18.

18. Wolff AC, Pesut A, Regan S. New graduate nurse practice readiness: Perspectives on the context shaping our understanding and expectations. Nurs Educ Today 2010;30:187191.

19. Baumberger-Henry M. Registered nurses' perspectives on the new graduate working in the emergency department or critical care unit. J Contin Educ Nurs 2012;43(7):299_ 305.

20. Freeling M, Parker S. Exploring experienced nurses' attitudes, views and expectations of new graduate nurses: A critical review. Nurse Educ Today 2015;35(2):e42-e49.

21. Lofmark A, Smide B, Wikblad K. Competence of newly-graduated nurses - A comparison of the perceptions of qualified nurses and students. J Adv Nurs 2006;53(6):721-728.

22. Hickey MT. Preceptor perceptions of new graduate readiness for practice. J Nurses Staff Dev 2009;25(1):35-41.

23. Hartigan I, Murphy S, Flynn AV, Walshe N. Acute nursing episodes which challenge graduate's competence: Perceptions of registered nurses. Nurse Educ Pract 2010;10:291-297.

24. Clark T, Holmes S. Fit for practice? An exploration of the development of newly qualified nurses using focus groups. Int J Nurs Stud 2007;44:1210-1220.

25. Hegney D, Eley R, Francis K. Queensland nursing staffs' perceptions of the preparation for practice of registered and enrolled nurses. Nurs Educ Today 2012;33(10):1148-1152

26. Walker A, Earl C, Costa B, Cuddihy L. Graduate nurses' transition and integration into the workplace: A qualitative comparison of graduate nurses' and nurse unit managers' perspectives. Nurse Educ Today 2013;33:291-296.

27. Chernomas, Care WD, McKenzie JL, Guse $L$, Currie J. 'Hit the ground running': Perspectives of new nurses and nurse managers on role transition and integration of new graduates. Nursing Leadersh 2010;22(4):70-87.

28. Crist JD, Tanner CA. Interpretation/analysis methods in hermeneutic interpretive phenomenology. Nurs Res 2003;52(3):202-5.

29. Lydon C. Burke E. Students experiences of theatre allocations. J Perioper Pract 2012:22(2):45-49.

30. Burns I, Paterson IM. Clinical practice and placement support: Supporting learning in practice. Nurse Educ Pract 2005;5(1):3-9.

31. Warne $T$, Johansson U, Papastavrou E An exploration of the clinical learning experience of nursing students in nine European countries. Nurse Educ Today 2010;30(8):809-815.

32. Schulz KE, DiSanto K. Investing in the future - an OR orientation program for nursing students. AORN J 1999;69:635-638.

33. Callaghan A. Student nurses' perceptions of learning in a perioperative placement. J Adv Nurs 2010;67(4):854-864.

34. Foran P. Undergraduate surgical nursing preparation and guided operating room experience: A quantitative analysis. Nurs Educ Pract 2015. Article in press doi:10.1016/j. nepr.2015.08.005.

35. Young LE. Mentoring new nurses in stressful times. Can Oper Room Nurs J 2009;27(2):6-7, 14-15, 29-30.

36. Pupkiewicz J, Kitson A, Perry J. What factors within the perioperative environment influence the training of scrub nurses? Nurse Educ Pract 2015;15(5):373-380.

37. Laschinger HKS. Job and career satisfaction and turnover intentions of newly graduated nurses. J Nurs Manag 2012;20(4):472-484.

38. Jacob J. Occupational stress of scrub/scout practitioner: Overview of selected literature. ACORN J 2015;28(3):15- 21.

39. Kingdon $B$, Halvorsen F. Perioperative nurses' perceptions of stress in the workplace. AORN J 2006;84(4):607-612, 614.

40. Taylor \& Ellison 1994 in Jacob J. Occupational stress of scrub/scout practitioner: Overview of selected literature. ACORN J 2015;28(3):15-21.

41. Higgins B, Maclntosh J. Operating room nurses' perceptions of the effects of physician-perpetrated abuse. Int Nurs Rev 2010;57(3):321-327.

42. Cook JK, Green M, Topp RV. Exploring the impact of physician verbal abuse on perioperative nurses. AORN J 2001;74(4):317$318,320,322-327,329-331$.

43. Mental health and wellbeing at the workplace - Protection and inclusion in challenging times [Internet]. Geneva: World Health Organization; 2010 [cited 2015 December 21]. Available from www.euro. who.int/data/assets/pdffile/ $\overline{0018 / 124047 / ~}$ e94345.pdf.

44. Longo J, Sherman RO. Leveling horizontal violence. Nurs Manage 2007;38(3):34-37, 50-51.

45. Shenton AK. Strategies for ensuring trustworthiness in qualitative research projects. Educ Inform 2004;22(2):63-75. 